\title{
Manejo del Cáncer de Vejiga mediante cirugía convencional en Solca, experiencia 2012-2017.
}

\section{Bladder Cancer Management through conventional surgery in Solca, experience 2012-2017. \\ Francisco de la Rosa Villao', Raúl Ibarra BurbanoiD, María Daniela Espinoza ${ }^{2}$, Xavier Wong Achi², Paul Ulloa Ochoa'2.}

\section{*Correspondencia:}

santiaguo_182@hotmail.com

Teléfono [593] 996752478

Conflicto de intereses: Los autores declaran no tener conflictos de intereses.

Fondos: Ver la página 216

Recibido: 1 Enero 2018

Aceptado: 11 Agosto 2018

Publicado: 30 Diciembre 2018

Membrete bibliográfico:

De la Rosa-Villao F, Ibarra R, Espinoza $\mathrm{M}$, Wong $\mathrm{X}$, Ulloa $\mathrm{P}$. Manejo del Cáncer de Vejiga mediante cirugía convencional en Solca, experiencia 2012-2017. Rev. Oncol. Ecu 2018;28(3):210218.

DOI: https://doi.org/10.33821/253

Copyright De la Rosa, et al. Este artículo es distribuido bajo los términos de Creative

Commons Attribution License, el cual permite el uso y redistribución citando la fuente y al autor original.
1. Instituto Oncológico Nacional "Dr. Juan Tanca Marengo", Solca-Guayaquil, Servicio de Cirugía General-Urología.

2. Postgrado de Cirugía General de la Universidad de Especialidades "Espíritu Santo" (UESS). Samborondón, Ecuador.

\section{Resumen}

Introducción: El cáncer de vejiga es la más común de las patologías del tracto urinario. La importancia del estudio radica en el manejo quirúrgico de los pacientes con este tipo de neoplasias vesicales que en ciertos casos con diagnóstico temprano suelen ser curativo. La técnica quirúrgica empleada de acuerdo al tipo histológico de los pacientes con cáncer de vejiga de este reporte fue el objetivo principal de este estudio.

Métodos: El presente es un estudio retrospectivo, fue realizado desde enero 2012 a julio 2017 en el que se revisaron las historias clínicas de los pacientes con diagnóstico de cáncer de vejiga atendidos en el Servicio de Urología Oncológica del Instituto Oncológico Nacional "Dr. Juan Tanca Marengo", Solca-Guayaquil. Se incluyeron pacientes operados dentro de la institución, con diagnóstico de cáncer de vejiga a quienes se les realizó Cistectomía radical más Bricker o Neovejiga. Se excluyeron pacientes con cáncer de vejiga oncológicamente no operables, casos clínicos con falta de información completa en la historia clínica, con exámenes diagnósticos incompletos. Se realizó un análisis estadístico descriptivo con porcentajes.

Resultados: Ingresaron al estudio 89 casos, 53 (59.6 \%) fueron hombres, 16 pacientes (18\%) con Cáncer de Vejiga de Bajo Grado Infiltrante a Lámina Propia (CABILAPRO), 1 paciente $(1.1 \%)$ con Cáncer de Vejiga de Bajo Grado Infiltrante a Muscular (CABIMUS), 5 pacientes (5.6\%) con Cáncer de Vejiga de Alto Grado Infiltrante a Lamina Propia (CALAPRO) y 63 pacientes $(70.8 \%)$ con Cáncer de Vejiga de Alto Grado Infiltrante a Muscular (CALMUS), Cáncer de Vejiga de Alto Grado Infiltrante a Grasa Perivesical 3 (3.4\%), de los cuales presentaron recidiva local pos tratamiento por CALMUS 13 
(14.6\%), 1 rabdosarcoma. Tratamiento quirúrgico del CALMUS 18 cirugías con derivación Bricker y 7 Neo-vejigas orto tópicas. Se realizaron RTUv en 50 pacientes (56.2 \%).

Conclusión: La cirugía de cistectomía más derivación Bricker se realizó en menos de un cuarto de la población estudiada, la cirugía RTUv fue la más prevalente.

Palabras Claves: VEJIGA URINARIA, CISTECTOMÍA, NEOPLASIAS DE LA VEJIGA URINARIA.

DOI: $10.33821 / 253$

\section{Abstract}

Introduction: Bladder cancer is the most common of the pathologies of the urinary tract. The importance of the study lies in the surgical management of patients with this type of bladder neoplasms that in certain cases with early diagnosis are usually curative. The surgical technique used according to the histological type of patients with bladder cancer in this report was the main objective of this study.

Methods: This is a retrospective study, carried out from January 2012 to July 2017, in which the clinical histories of patients diagnosed with bladder cancer treated at the Oncology Urology Service of the National Oncological Institute "Dr. Juan Tanca Marengo ", Solca-Guayaquil. Patients operated on within the institution were included, with diagnosis of bladder cancer who underwent radical cystectomy plus Bricker or Neovejiga. Patients with oncologically inoperable bladder cancer were excluded, clinical cases with lack of complete information in the clinical history, with incomplete diagnostic tests. A descriptive statistical analysis was performed with percentages.

Results: 89 cases were included in the study, $53(59.6 \%)$ were men, 16 patients (18\%) with Low-Grade Infiltrating Bladder Cancer (CABILAPRO), 1 patient (1.1\%) with Low-grade Bladder Cancer Infiltrant to Muscular (CABIMUS), 5 patients (5.6\%) with High-Grade Infiltrating Bladder Laminal Cancer (CALAPRO) and 63 patients (70.8\%) with High-Grade Infiltrating Muscle Bladder Cancer (CALMUS), Cancer High Grade Infiltrant to Perivesical Fat Bladder 3 (3.4\%), of which local recurrence after treatment by CALMUS $13(14.6 \%)$, 1 rhabdosarcoma. Surgical treatment of CALMUS 18 surgeries Bricker and $7 \mathrm{Neo}$-bladders orthotopic. TURb was performed in 50 patients (56.2\%).

Conclusion: Bricker surgery was performed in less than a quarter of the study population, TURb surgery was the most prevalent.

Keywords: URINARY BLADDER, CISTECTOMY, NEOPLASIAS OF THE URINARY BLADDER.

DOI: $10.33821 / 253$ 


\section{Introducción}

Según la Sociedad de Lucha Contra el Cáncer (SOLCA) los valores estadísticos con respecto a los tumores del tracto urinario fueron 1.6 en mujeres y 4.4 en hombres para el registro del 2016 [1].

El cáncer de vejiga es la más común de las patologías malignas del tracto urinario. La tasa mundial estandarizada por edad es de 10.1 por 100 mil en hombres y de 2.5 por 100 mil en mujeres [2]. En Europa, la incidencia más alta de cáncer de vejiga (tasa estandarizada por edad) aparece en Europa occidental (23.6 en hombres y 5.4 en mujeres) y en la región del sur (27.1 en hombres y 4.1 en mujeres), seguido por el norte de Europa (16.9 en hombres y 4.9 en mujeres). La incidencia más baja se ha observado en las regiones de Europa oriental (14.7 en hombres y 2.2 en mujeres) [2]. El cáncer de vejiga es el quinto en frecuencia entre los hombres en países desarrollados (tras los tumores de próstata, pulmón, colorectales y de estómago), con aproximadamente 357 mil nuevos casos al año en todo el mundo (274 mil hombres y 83 mil mujeres) [3]. El cáncer de vejiga es aproximadamente 4 veces más frecuente en hombres que en mujeres. Sin embargo, al momento del diagnóstico, las mujeres tienen cáncer de vejiga avanzado con más frecuencia [4].

El cáncer de vejiga es un tumor de histología predominantemente urotelial. El consumo de Tabaco es el factor etiológico que más impacto tiene en el desarrollo del mismo [5]. La hematuria se considera el signo más común en este tipo de neoplasias. El diagnóstico de cáncer de vejiga depende del examen cistoscópico y la evaluación histológica del tejido resecado [6].

El diagnóstico de cáncer in situ se hace utilizando la combinación de cistoscopia, citología urinaria y evaluación histológica de biopsias de vejiga múltiples. El informe patológico debería especificar el grado de la lesión, la profundidad de la invasión tumoral en la pared vesical y si la lámina propia y el músculo están presentes en la muestra. Una Resección Transuretral Vesical (RTUv) completa y correcta es esencial para el pronóstico del paciente [2].

La radioterapia neo-adyuvante ha demostrado que va mejor en los tumores $\mathrm{t} 3 \mathrm{~b}$, la cirugía estándar en los pacientes varones con neoplasias de vejiga incluye la extirpación de toda la vejiga, próstata, vesículas seminales, uréteres distales (longitud del segmento sin definir) y los ganglios linfáticos correspondientes [7].

La cistectomía radical laparoscópica con linfadenectomía y derivación urinaria es una cirugía de empleo creciente. Se necesitan estudios que avalen la efectividad oncológica y la seguridad de este abordaje mínimamente invasivo [8].

Por ello, al realizar una técnica exigente de cistectomía resulta mandatorio conseguir márgenes negativos y un elevado número de ganglios pélvicos extirpados [8] Con el manejo quirúrgico agresivo del cáncer de vejiga invasivo se puede lograr una excelente supervivencia a largo plazo con una baja incidencia de recurrencia pélvica [9].

La primera reconstrucción vesical ortotópica en humanos fue descrita por Lemoine en 1913. En 1979, Camey y Le Duc informaron su experiencia en la creación de una neovejiga 
con intestino delgado, con la preservación del esfínter uretral durante la cistectomía [5]. La reconstrucción del tracto urinario tras la cistectomía radical ha evolucionado desde la simple derivación urinaria hasta la reconstrucción anatómica y funcional del mismo lo más próxima posible al estado preoperatorio del paciente, con objeto de proteger y conservar la función del tracto urinario superior [7].

Los autores deciden presentar este reporte debido a la importancia de la cirugía para tratamiento del cáncer de vejiga con la formación de nuevos continentes urinarios para mantener la fisiología urinaria del paciente y darle un mejor estilo de vida.

\section{Materiales y Métodos}

El presente es un estudio retrospectivo, descriptivo realizado desde enero 2012 a julio 2017 en el que se revisaron las historias clínicas de todos los pacientes con diagnóstico de cáncer de vejiga, atendidos en el Servicio de Urología Oncológica del Instituto Oncológico Nacional "Dr. Juan Tanca Marengo", Solca-Guayaquil. Se incluyeron pacientes operados dentro de la institución, con diagnóstico de cáncer de vejiga a quienes se les realizó Cistectomía radical más derivación tipo Bricker o Neovejiga. Se excluyeron pacientes con cáncer de vejiga oncológicamente no operables, casos clínicos con falta de información completa en la historia clínica, con exámenes diagnósticos incompletos. Se realizó un análisis estadístico descriptivo con porcentajes.

\section{Resultados}

Ingresaron al estudio 89 casos, 53 (59.6\%) fueron hombres y 36 (40.4\%) mujeres, se encontró que 16 pacientes (18\%) presentaron Cáncer de Vejiga de Bajo Grado Infiltrante a Lámina Propia (CABILAPRO), 1 paciente $(1.1 \%)$ con Cáncer de Vejiga de Bajo Grado Infiltrante a Muscular (CABIMUS), 5 pacientes (5.6 \%) con Cáncer de Vejiga de Alto Grado Infiltrante a Lamina Propia (CALAPRO) y 63 pacientes (70.8\%) con Cáncer de Vejiga de Alto Grado Infiltrante a Muscular (CALMUS),Cáncer de Vejiga de Alto Grado Infiltrante a Grasa Perivesical 3 (3.4\%), de los cuales presentaron recidiva local pos tratamiento por CALMUS $13(14.6 \%), 1$ rabdosarcoma (Figura 1).

En el estudio se obtuvieron como tratamiento quirúrgico del CALMUS 15 Cistectomías radicales más derivación tipo Bricker y 7 neo vejigas orto tópicas de las cuales 4 son funcionales actualmente y 3 terminaron en Cistectomías radicales más derivación tipo Bricker debido a complicaciones isquémicas por las cuales fueron reintervenidos dando como total 18 Bricker (20.1\%) y 4 Neovejigas ( $4.5 \%$ ), de los 89 pacientes se realizaron RTUv en 50 pacientes (56.2\%) la cual fue diagnóstica y terapéutica, con buenos resultados en especial en CABILAPRO-CABIMUS-CALAPRO (Figura 2). 
Figura 1. Anatomía patológica de los pacientes con Ca vesical

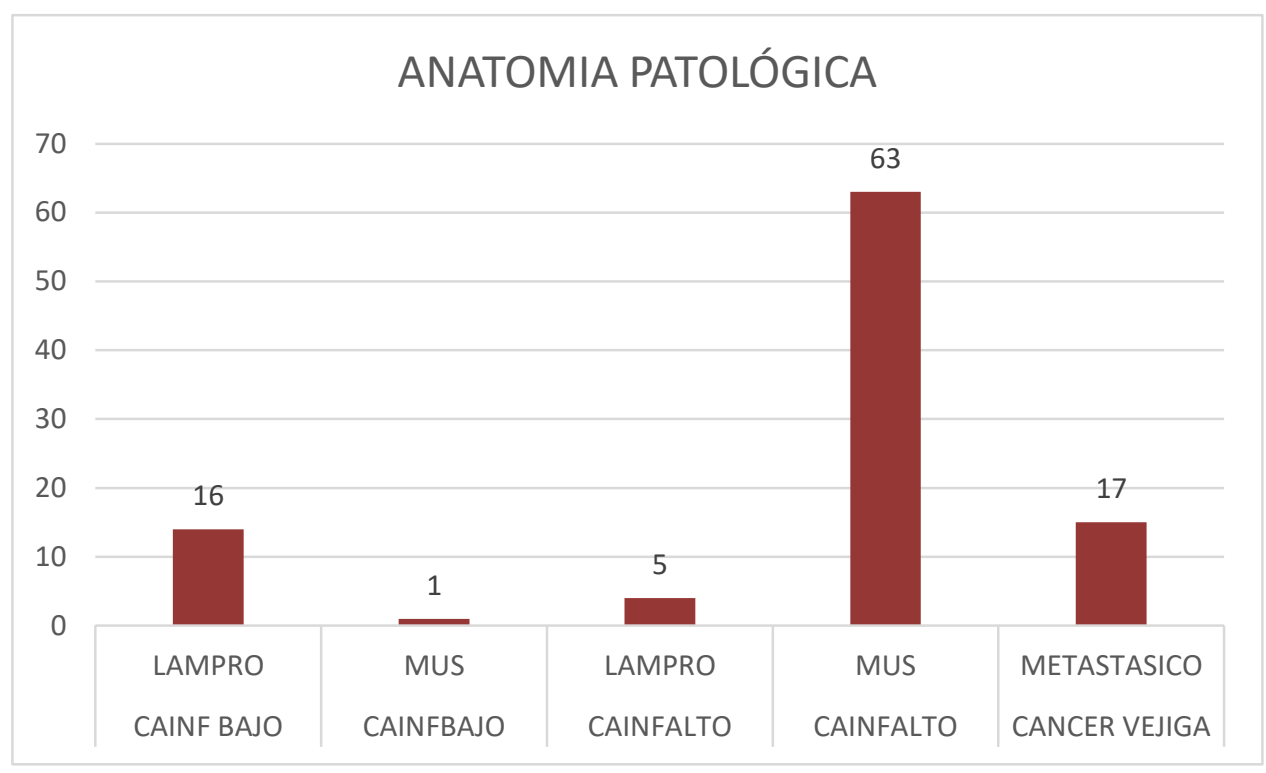

Anatomía Patológica resultados frecuencia de diagnósticos, los 4 restantes son canceres de vejiga infiltrantes a grasa perivesical y 1 rabdosarcoma.

Figura 2. Pacientes operados de Neovejiga y Bricker

\section{PACIENTES OPERADOS}

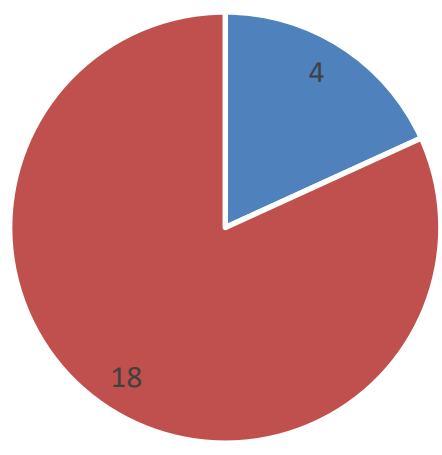

- PACIENTES OPERADOS NEOVEJIGA - PACIENTES OPERADOS BRIQUER 


\section{Discusión}

En una serie retrospectiva de 153 pacientes, con una clara indicación para la cirugía radical de vejiga por cáncer localmente avanzado, encontraron que los pacientes tratados durante más de 90 días después del diagnóstico primario mostraron un aumento significativo en enfermedad extra vesical ( 81 vs. $52 \%$ ). El retraso en la cistectomía afecta el resultado del tratamiento y el tipo de derivación urinaria (el retraso en la cistectomía se asocia más con derivaciones con conducto ileal) [10].

En los tumores no músculo infiltrante y en función de los factores pronósticos se establecen Grupos de riesgo (bajo, medio y alto) que determinan el mejor tratamiento posible. En los casos de bajo Riesgo es suficiente la administración de una dosis posoperatoria de quimioterapia intravesical, en riesgo intermedio el paciente debe recibir quimioterapia o inmunoterapia intravesical adyuvante. En los casos de alto riesgo se recomiendan la inmunoterapia intravesical durante un periodo de uno a tres años. La afectación del músculo detrusor tiene implicaciones pronósticos y de tratamiento. En el caso de los tumores músculo infiltrantes, la cirugía con quimioterapia neoadyuvante es el tratamiento estándar. En los tumores metastáticos la quimioterapia basada en cisplatino es el tratamiento de elección [5].

La quimioterapia neoadyuvante también tiene desventajas potenciales: a) los pacientes sin enfermedad micrometastásica de los cuales aproximadamente la mitad son pacientes clínicamente NO MO recibirán tratamiento innecesario; b) los errores en la estatificación pueden hipotéticamente llevar a un exceso de tratamiento; c) la cistectomía diferida puede comprometer el resultado en los pacientes que no responden a la quimioterapia d) la quimioterapia puede tener efectos secundarios que afectan al resultado de la cirugía y al tipo de derivación urinaria [6].

En el presente estudio se realizó cirugía ya que es el tratamiento estándar del cáncer de vejiga. Las contraindicaciones relativas específicas de una neovejiga ortotópica son altas dosis de RT preoperatoria, enfermedad de estenosis uretral compleja e incontinencia severa relacionada con el esfínter uretral [6].

En el caso de la neo vejiga en el manejo postoperatorio a los 10 días, se realiza una cistografía para asegurar la estanqueidad de la neovejiga. Tras esta prueba se puede retirar el catéter de cistotomía y a las 48 horas la sonda uretral [7]. En la casuística presentada se empleó el mismo manejo para sonda y tutores durante el postoperatorio.

La cistectomía radical laparoscópica con linfadenectomía y derivación urinaria es una cirugía de empleo creciente. Se necesitan estudios que avalen la efectividad oncológica y la seguridad de este abordaje mínimamente invasivo [8]. Por tanto no se usó esta opción de tratamiento en la presente casuística.

Por ello, al realizar una técnica exigente de cistectomía resulta mandatorio conseguir márgenes negativos y un elevado número de ganglios pélvicos extirpados [8]. Con el manejo quirúrgico agresivo del cáncer de vejiga invasivo se puede lograr una excelente supervivencia a largo plazo con una baja incidencia de recurrencia pélvica [9]. 
La importancia del estudio radica en el manejo quirúrgico de los pacientes con este tipo de neoplasias vesicales, el cual en mucho de los casos suele ser curativo.

\section{Conclusiones}

La importancia del manejo quirúrgico en los cánceres de vejiga de alto y bajo grado, están representados por la RTUv para diagnóstico y tratamiento con tasa alta de curación si el diagnóstico es oportuno. La Cistectomía radical más derivación tipo Bricker en Cáncer de techo o en neovejigas no funcionales o complicaciones isquémicas y la cistectomía radical más neovejiga ortotópica para cáncer de piso vesical siendo este último el procedimiento que le devuelve la fisiología al aparato urinario. La cistectomía radical en cáncer de vejiga de alto grado con o sin infiltración a muscular no debe retrasarse ya que se ha demostrado el aumento de la supervivencia de los pacientes sometidos a cistectomía en relación a los pacientes sometidos a tratamiento conservador.

\section{Agradecimientos}

Reconocemos a las personas que participaron indirectamente en el estudio tales como el personal técnico, pacientes y personal del Instituto Oncológico Nacional “Dr. Juan Tanca Marengo", Solca-Guayaquil.

\section{Información adicional}

Nota del Editor

La Revista Oncología Ecu permanece neutral con respecto a los reclamos jurisdiccionales en mapas publicados y afiliaciones institucionales.

\section{Abreviaturas}

CABILAPRO: Cáncer de Vejiga de Bajo Grado Infiltrante a Lámina Propia.

CABIMUS: Cáncer de Vejiga de Bajo Grado Infiltrante a Muscular.

CALAPRO: Cáncer de Vejiga de Alto Grado Infiltrante a Lamina Propia.

CALMUS: Cáncer de Vejiga de Alto Grado Infiltrante a Muscular.

RTUv: Resección Transuretral Vesical.

\section{Archivos Adicionales}

Ninguno declarado por los autores.

\section{Fondos}

Los fondos de la investigación fueron propios de los autores del presente artículo. 


\section{Disponibilidad de datos y materiales}

Existe la disponibilidad de datos bajo solicitud al autor de correspondencia. No se reportan otros materiales.

\section{Contribuciones de los autores}

FDLRV, RIB, realizaron la idea de investigación, revisión bibliográfica. MDE, XWA, PUO, recolección de datos, escritura del artículo. FDLRV, RIB realizó el análisis crítico del artículo. $\mathrm{RIB}$, realizó las correcciones editoriales. Todos los autores leyeron y aprobaron la versión final del artículo.

Aprobación de ética y consentimiento para participar

No aplica a este estudio.

\section{Consentimiento para publicación}

No aplica.

\section{Información de los autores}

Francisco de la Rosa Villao, Médico Cirujano General y Urólogo, del Servicio de Instituto Oncológico Nacional "Dr. Juan Tanca Marengo", Solca-Guayaquil, Servicio de Cirugía General-Urología.

Raúl Ibarra Burbano, Postgrado de Cirugía General de la Universidad de Especialidades "Espíritu Santo" (UESS). Samborondón, Ecuador. R2 posgrado Cirugía Solca-Guayaquil. https://orcid.org/0000-0002-9423-0784

María Daniela Espinoza, Postgrado de Cirugía General de la Universidad de Especialidades "Espíritu Santo" (UESS). Samborondón, Ecuador. R2 posgrado Cirugía Solca-Guayaquil.

Xavier Wong Achi, Postgrado de Cirugía General de la Universidad de Especialidades "Espíritu Santo" (UESS). Samborondón, Ecuador. R1 posgrado Cirugía Solca-Guayaquil.

Paul Ulloa Ochoa, Postgrado de Cirugía General de la Universidad de Especialidades "Espíritu Santo" (UESS). Samborondón, Ecuador. R3 posgrado Cirugía Solca-Guayaquil. 
Acceda a la revisión de pares académicos en el siguiente enlace: https://publons.com/review/3828495

\section{Referencias}

Abreviaturas en la referencias

DOI: Digital Object

Identifier

PMID: PubMed Identifier

SU: Short URL
1. SOCIEDAD ONCOLOGICA DE LUCHA CONTRA EL CANCER (SOLCA). Estadísticas de cáncer en Guayaquil-Ecuador. Registro de Tumores Guayaquil. 2017.

2. Babjuk M, Oosterlinck W, Sylvester R, Kaasinen E, Böhle A, Palou J, et al. Guía clínica del carcinoma urotelial de vejiga no músculo-invasivo de la Asociación Europea de Urología. Actualización 2011. Actas Urol Esp. 2012;36(7):389-402. DOI: 10.1016/j.acuro.2011.12.001

3. Razmaria A. Bladder Cancer. Rev JAMA. 2015;314(17):1886. DOI:10.1001/jama.2015.13738

4. Martínez A, Hernández N, Quintero J, Flores D, Moreno J, Vázquez E. Trece años de experiencia en el manejo de cáncer vesical con neovejiga ortotópica de Studer. Rev Cirugía y Cirujanos. 2009; 77(6):443450. SU: goo.gl/1SH7bN

5. Ríos E, Jiménez AM. Cáncer de Vejiga. Rev Medicine 2017;12(33):1955-1965. DOI: 10.1016/j.med.2017.04.020

6. Stenzl A, Cowan NC, De Santis M, Kuczyk MA, Merseburger AS, Ribal MJ, et al. Tratamiento del cáncer de vejiga con invasion muscular y metastásico: actualización de la Guía Clínica de la EAU. Actas Urol Esp. 2012;36(8):449-460. DOI: 10.1016/j.acuro.2011.11.001

7. Álvarez $\mathrm{M}$, Llorente $\mathrm{C}$. Manejo perioperatorio y resultados en pacientes con neovejiga ileal ortotópica. Actas Urol Esp. 2008;32(3):297-306. DOI: 10.1016/S0210-4806(08)73834-8

8. Espinas C, Alonso JM, Mateo E, Martín AM, Dorado JF, Arance I, et al. Estudio prospectivo comparativo entre cistectomia radical y laparoscopica abierta: resultados operatorios y oncologicos. Actas Urol Esp. 2018;42(2):94-102. DOI: 10.1016/j.acuro.2017.04.004

9. Stein JP, Lieskovsky G, Cote R, Groshen S, Feng AC, Boyd S, et al. Radical cystectomy in the treatment of invasive bladder cancer: long-term results in 1,054 patients. J Clin Oncol 2001;19(3):666. DOI: $10.1200 / J C 0.2001 .19 .3 .666$

10. Citarella D, Quiroga W, Fernández F, Patiño I, Estrada A. Guia de manejo en cáncer vesical.Rev Urol Col 2016;25(2):154-168. SU: goo.gl/mqT916 